\title{
EFECTOS SOBRE LA AUTOPERCEPCIÓN EN PERSONAS MAYORES DE 60 AÑOS DE UN PROGRAMA DE ACTIVIDAD FÍSICA EN EL AGUA
}

PROGRAMA DE ACTIVIDAD FÍSICA EN EL AGUA. EFFECTS ON SELF-PERCEPTION IN PEOPLE OVER 60 YEARS OF A PROGRAM OF PHYSICAL ACTIVITY IN WATER

Serrano-Huete, V. ${ }^{1}$, Lozano-Aguilera, E. ${ }^{2}$, Terán-Torres, M. C. ${ }^{3}$, Laredo-Aguilera, JA. ${ }^{4}$ y Párraga-Montilla, J. A. ${ }^{5}$

1 V. Serrano-Huete. Doctor. Universidad de Jaén, España. Autor de correspondencia. victorsh1981@hotmail.com.

${ }^{2}$ E. Lozano-Aguilera. Universidad de Jaén. Profesor titular. Universidad de Jaén, España. elozano@ujaen.es

${ }^{3}$ M. C. Terán-Torres. Universidad de Jaén. Doctor. Universidad de Jaén, España. cristeran@hotmail.com

4 J. A. Laredo-Aguilera. Universidad de Jaén. Doctor. Universidad de Castilla la Mancha, España. jalaredo@hotmail.com

5 J. A. Párraga-Montilla. Universidad de Jaén. Profesor titular. Universidad de Jaén, España. jparraga@ujaen.es

Código UNESCO: 6108. Psicología de la vejez

Consejo de Europa: 15. Psicología del deporte

DOI: http://dx.doi.org/10.24310/riccafd.2018.v7i2.5098

\section{RESUMEN}

Introducción: se observa la incidencia del proceso de envejecimiento sobre las capacidades físicas, mentales y sociales y su relación con la calidad de vida, de ahí la importancia de la práctica de actividad física en mayores. El medio acuático es un aliado en la actividad física con mayores por aplicar un bajo impacto articular. Objetivo: comprobar la evolución del estado de ánimo de los participantes en un programa de actividad física acuática. Método: 98 adultos $(69,08 \pm 7,39$ años) realizaron un programa de actividad física acuática con dos sesiones (60 min) semanales durante 20 semanas. Hubo tres evaluaciones: pre-intervención, durante y post-intervención, mediante la escala POMS y el cuestionario SF-36. Resultados: se encontraron diferencias significativas, con aumento del vigor, reducción de la tensión, fatiga, depresión y hostilidad, percibiendo una mejora de salud. Conclusión: la actividad física acuática mejora la 
percepción del estado de ánimo de los participantes, tanto en el componente físico como en el mental.

Palabras clave: adulto mayor, envejecimiento activo, actividad física acuática, estado de ánimo, percepción de salud.

\begin{abstract}
Introduction: the incidence of the aging process is observed on the physical, mental and social capacities and their relationship with the quality of life, hence the importance of the practice of physical activity in the elderly. The aquatic environment is an ally in physical activity with adults for applying a low joint impact. Objective: to check the evolution of the mood of the participants in a program of aquatic physical activity. Method: 98 adults $(69,08 \pm 7,39$ years) performed a program of aquatic physical activity with two sessions (60 min) per week for 20 weeks. There were three evaluations: pre-intervention, during and post-intervention, using the POMS scale and the SF-36 scale. Results: significant differences were found, with increased vigor, reduction of tension, fatigue, depression and hostility, perceiving an improvement in health. Conclusion: aquatic physical activity improves the perception of the mood of the participants, both in the physical component and in the mental.
\end{abstract}

Key words: elderly, active aging, aquatic physical activity, mood, health percep-tion.

\title{
INTRODUCCIÓN
}

El envejecimiento se define como el cambio gradual e intrínseco en un organismo que conduce a un riesgo creciente de vulnerabilidad, pérdida de vigor, enfermedad y muerte (1). Es un de fenómeno de difícil manejo y solución técnicoeconómica para los países en desarrollo

Los cambios anatómicos y fisiológicos asociados al envejecimiento han sido íntimamente estudiados y descritos por varios autores, es por eso que sabemos que es un proceso dinámico y va en función de factores endógenos y exógenos de cada persona. Este declive orgánico produce alteración en los distintos sistemas, pudiéndose observar una disminución clara en sus funciones (2-5).

El concepto de envejecimiento activo, creado por la Organización Mundial de Salud en 1997, que tiene como base el principio de permitir a las personas mayores que permanezcan integradas y motivadas en la vida laboral y social (6). Así, los principales beneficios de la práctica regular de actividad física según la OMS (7) son la reducción del riesgo de hipertensión, cardiopatía coronaria, accidente cerebro vascular, diabetes, cáncer de mama y de colon, depresión y caídas, la mejora la salud ósea y funcional, siendo un determinante clave del gasto ener- 
gético, y es por tanto fundamental para el equilibrio calórico y el control del peso. Los principales beneficios de la práctica regular de actividad física según la OMS (8) son la reducción del riesgo de hipertensión, cardiopatía coronaria, accidente cerebro vascular, diabetes, cáncer de mama y de colon, depresión y caídas, la mejora la salud ósea y funcional, siendo un determinante clave del gasto energético, y es por tanto fundamental para el equilibrio calórico y el control del peso.

Uno de los instrumentos más utilizados para valorar la calidad de vida es el SF-36, que es un instrumento muy adecuado para su uso en investigación y en la práctica clínica. Las propiedades psicométricas están altamente demostradas en el estudio Una década de experiencia y nuevos desarrollos (9), donde se muestran las excelentes propiedades psicométricas de dicho cuestionario. Fueron tomados datos de fiabilidad (consistencia interna y reproducibilidad), el modelo de medida, la validez del constructo y la sensibilidad al cambio, la cual engloba categorías de estado de salud física, salud mental y salud general.

El agua es un medio poco habitual en el desarrollo de las actividades cotidianas de las personas, no es el medio natural donde nos desenvolvemos, sin embargo en la actualidad es cada día más importante para todos los segmentos de la población (10). La práctica de ejercicio en el medio acuático para personas mayores está indicado para mejorar y mantener la capacidad de movimiento por los pocos riesgos que comporta y sus amplios beneficios $(10,11)$, dado que en este medio se rompen las barreras existentes, entre ellas el miedo de caer, el dolor, la incomodidad y la falta de forma física (12), abriendo así una alternativa al ejercicio para aquellos adultos mayores que no podían realizar ejercicio en tierra, como consecuencia de la fuerza de la gravedad y el propio peso corporal, que se ven compensados en las actividades físicas acuáticas.

El objetivo de este trabajo es comprobar la evolución del estado de ánimo de los participantes en un programa de actividad física acuática de 20 semanas de duración, valorando la calidad de vida percibida. Así, podemos hipotetizar que la práctica de actividad física en el medio acuático incidirá positivamente en el estado de ánimo y la calidad de vida de las personas mayores de 60 años.

\section{MATERIAL Y MÉTODO}

\section{Participantes:}

98 participantes, 31 hombres y 67 mujeres (74,3\% y 25,7\% respectivamente), con una media de edad de 69,08 $( \pm 7,39)$ años, $150,95( \pm 8,66) \mathrm{cm}$ de altura y un peso de 64,407 $( \pm 10,14) \mathrm{kg}$ e IMC de 28,28 $( \pm 4,2) \mathrm{Kg} / \mathrm{m}^{2}$. En la realización del estudio se siguieron las recomendaciones éticas aportadas por la $64^{a}$ Asamblea General, Fortaleza, Brasil (octubre 2013). Se obtuvo un informe favorable del comité de bioética de la Universidad de Jaén. Los participantes firmaron un consentimiento de participación voluntaria, en el que se les informó de las características de la investigación. 


\section{Diseño:}

El entrenamiento acuático se basó en las recomendaciones de actividad física de la ACMS (13) y en las recomendaciones para la realización de actividad física acuática $(10,12)$, creando una rutina multicomponente.

10: semana de adaptación. 2 sesiones. $90 \mathrm{~min} / \mathrm{sesión.}$

2: 5 semanas. 2 sesiones semanales. $60 \mathrm{~min} /$ sesión. 60 - 80\% frecuencia cardíaca máxima. Cada sesión tuvo el siguiente esquema:

Toma de contacto.

Calentamiento, activación cardiovascular, amplitud de movimiento (15 min.)

Parte principal: trabajo de contenidos marcados (35 min.)

Vuelta a la calma: relajación, flexibilidad (10 min.).

\section{Materiales e instrumentos}

Se utilizaron los cuestionarios POMS29 (14) y el SF-36v2 (9) para el registro de los datos.

\section{Procedimiento:}

Se convocó en cada casa comunal a la población susceptible de estudios de los municipios de la ciudad de Quito (Ecuador), implicados a una reunión explicativa de los procedimientos y objetivos del presente estudio. Tras ella, se reclutó aquellos usuarios que desearon participar, previa firma de consentimiento informado y evaluación médica. Se realizaron posteriormente los test POMS y SF-36v2, para las tres tomas de datos.

Los 98 participantes fueron divididos en seis grupos de manera aleatoria, cuatro grupos de 16 y dos de 17 personas, de los cuales se subdividieron en dos grupos, que trabajaron martes y jueves y los otros miércoles y viernes, asignándole una hora a cada grupo. Iniciamos con una la toma de contacto, donde se explicaba la dinámica de trabajo de ese día, seguido por el estiramiento inicial y calentamiento que tuvo una duración de 15 minutos. Continuamos con la parte de acondicionamiento, donde se trabajó con material auxiliar tablas, tubos de flotación o pelotas, su duración fue de 35 minutos. Para terminar se procedió a la fase de relajación y estiramiento de grandes grupos musculares (10 minutos), y se motivó y alentó a los participantes durante la práctica. Tras 10 semanas de entrenamiento, se procedió a la evaluación intermedia, finalizándose con una última evaluación al concluir las 20 semanas de entrenamiento.

\section{RESULTADOS}

\section{Bateria POMS}

Los resultados de la Batería POMS en las tres evaluaciones (pre, durante y post-tratamiento) vienen reflejados en la tabla 1 , mostrándose su posterior interpretación gráfica en la figura 1. 


\begin{tabular}{|c|c|c|c|c|c|}
\hline \multirow[t]{2}{*}{ VARIABLE } & \multirow[t]{2}{*}{ EVALUACIÓN } & \multirow[t]{2}{*}{ MEDIA } & \multirow[t]{2}{*}{ ERROR TIP } & \multicolumn{2}{|c|}{$\begin{array}{l}\text { INTERVALO DE CONFIANZA } \\
\text { AL } 95 \%\end{array}$} \\
\hline & & & & \begin{tabular}{|l} 
Límite \\
inferior
\end{tabular} & $\begin{array}{l}\text { Límite } \\
\text { superior }\end{array}$ \\
\hline \multirow{3}{*}{ FEDEPRE } & $1(2,3)$ & 2,716 & ,482 & 1,754 & 3,679 \\
\hline & $2(1)$ & 1,478 & ,260 & ,959 & 1,997 \\
\hline & $3(1)$ & 1,134 & ,258 & ,620 & 1,649 \\
\hline \multirow{3}{*}{ FTEN } & $1(2,3)$ & 4,565 & ,448 & 3,672 & 5,459 \\
\hline & $2(1,3)$, & 2,855 & ,329 & 2,199 & 3,511 \\
\hline & $3(1,2)$ & 1,638 & ,229 & 1,181 & 2,095 \\
\hline \multirow{3}{*}{ FFAT } & $1(2,3)$, & 4,091 & ,460 & 3,171 & 5,010 \\
\hline & $2(1)$ & 2,288 & ,344 & 1,601 & 2,975 \\
\hline & $3(1)$ & 1,939 & ,320 & 1,300 & 2,579 \\
\hline \multirow{3}{*}{ FVIG } & $1(2,3)$ & 11,986 & ,504 & 10,981 & 12,990 \\
\hline & $2(1,3)$ & 14,386 & ,517 & 13,354 & 15,417 \\
\hline & $3(1,2)$ & 17,000 & ,518 & 15,967 & 18,0333 \\
\hline \multirow{3}{*}{ FHOS } & $1(2,3)$ & 3,014 & ,429 & 2,159 & 3,870 \\
\hline & $2(1)$ & 1,290 & ,231 & 829 & 1,751 \\
\hline & $3(1)$ & ,899 & ,199 &, 501 & 1,296 \\
\hline
\end{tabular}

Tabla 1. Resultados de las tres evaluaciones para las distintas variables correspondientes a la Batería POMS. En la columna evaluación, distinto número indica diferencias significativas entre ambas, todas ellas menores de $p=0,050$. FDEPRE: factor de depresión, FFAT: factor de fatiga, FTEN: factor de tensión, FVIG: factor de vigor, FHOS: factor de hostilidad.

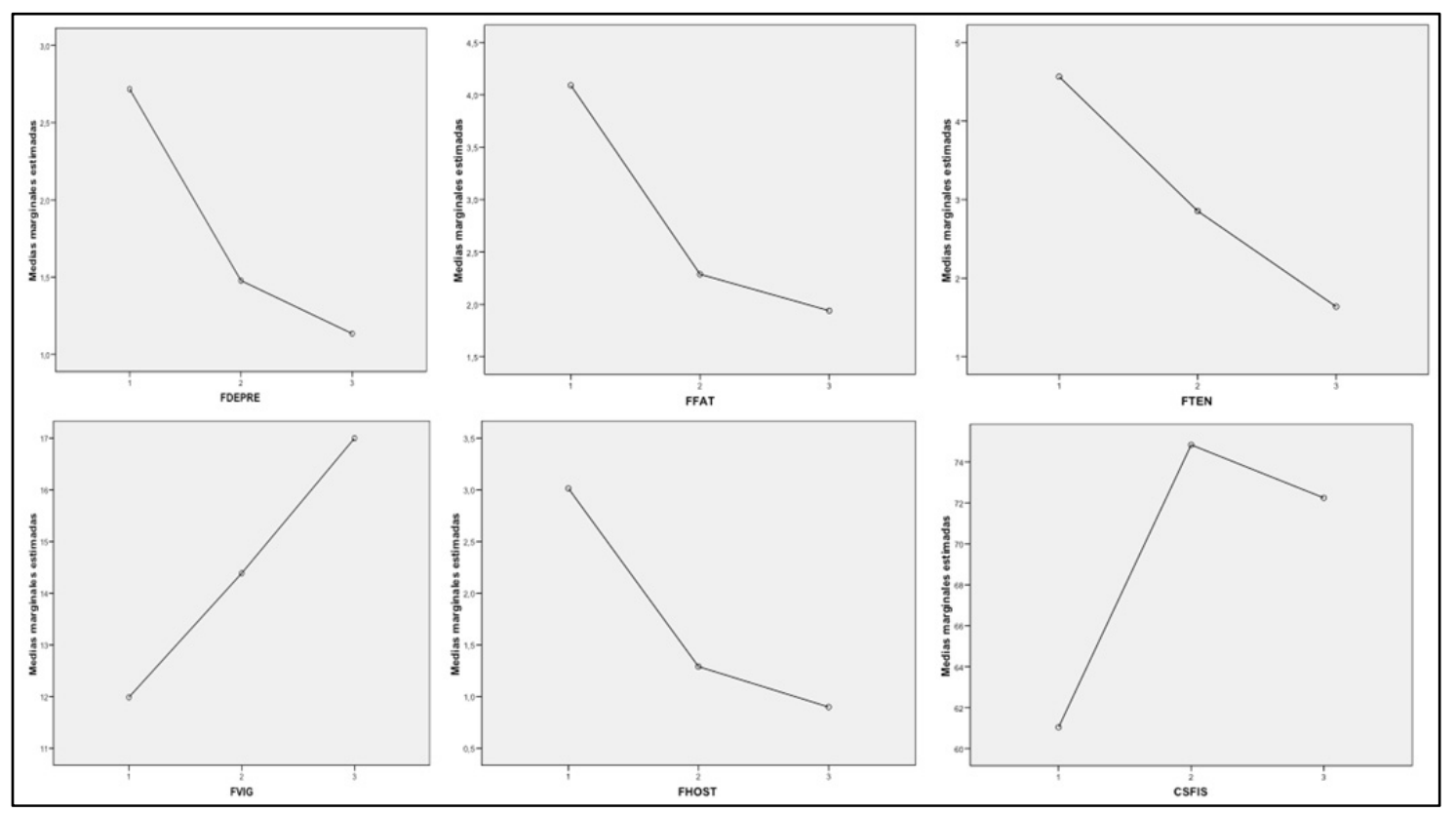

Figura 2. Representación gráfica de las tres tomas para las distintas variables correspondientes a la Batería POMS. FDEPRE: factor de depresión, FFAT: factor de fatiga, FTEN: factor de tensión, FVIG: factor de vigor, FHOS: factor de hostilidad. 


\section{Cuestionario SF-36v2}

Los resultados del cuestionario de salud SF-36v2 en sus tres tomas de datos (pre, durante y post-tratamiento) se muestran en la tabla 2. Por su parte, de forma gráfica se exponen dichos resultados en la figura 2.

\begin{tabular}{|c|c|c|c|c|c|}
\hline \multirow[t]{2}{*}{ VARIABLE } & \multirow[t]{2}{*}{ EVALUACIÓN } & \multirow[t]{2}{*}{ MEDIA } & \multirow[t]{2}{*}{ ERROR TIP } & \multicolumn{2}{|c|}{\begin{tabular}{|l|} 
INTERVALO DE \\
CONFIANZA AL 95\%
\end{tabular}} \\
\hline & & & & $\begin{array}{l}\text { Límite } \\
\text { inferior }\end{array}$ & $\begin{array}{l}\text { Límite supe- } \\
\text { rior }\end{array}$ \\
\hline \multirow{3}{*}{ CSFIS } & $1(2,3)$ & 61,043 & 2,435 & 56,184 & 65,901 \\
\hline & $2(1,3)$ & 74,828 & 2,017 & 70,804 & 78,853 \\
\hline & $3(2,3)$ & 72,240 & 2,122 & 68,007 & 76,473 \\
\hline \multirow{3}{*}{ CSM } & $1(2,3)$ & 62,041 & 2,174 & 57,703 & 66,379 \\
\hline & $2(1)$ & 79,184 & 1,763 & 75,667 & 82,701 \\
\hline & $3(1)$ & 76,665 & 1,939 & 72,796 & 80,534 \\
\hline \multirow{3}{*}{ CSTOT } & $1(2,3)$ & 61,436 & 2,141 & 57,165 & 65,706 \\
\hline & $2(1)$ & 76,643 & 1,802 & 73,049 & 80,238 \\
\hline & $3(1)$ & 74,002 & 1,921 & 70,171 & 77,834 \\
\hline \multirow{3}{*}{ DC } & $1(2,3)$ & 52,283 & 3,218 & 45,861 & 58,704 \\
\hline & $2(1,3)$ & 75,072 & 2,537 & 69,969 & 80,176 \\
\hline & $3(1,2)$ & 64,457 & 3,243 & 57,985 & 70,928 \\
\hline \multirow{3}{*}{ FF } & $1(2,3)$ & 58,297 & 3,069 & 52,174 & 64,420 \\
\hline & $2(1)$ & 69,783 & 2,792 & 64,212 & 75,354 \\
\hline & $3(1)$ & 67,464 & 3,041 & 61,396 & 73,531 \\
\hline \multirow{3}{*}{ RF } & $1(2,3)$ & 73,460 & 2,955 & 67,564 & 79,357 \\
\hline & $2(1)$ & 90,761 & 1,989 & 86,792 & 94,730 \\
\hline & $3(1)$ & 88,587 & 1,657 & 85,280 & 91,894 \\
\hline \multirow{3}{*}{ SG } & $1(2,3)$ & 58,607 & 2,384 & 53,852 & 63,363 \\
\hline & $2(1)$ & 70,464 & 1,968 & 66,537 & 74,391 \\
\hline & $3(1)$ & 71,452 & 2,028 & 67,406 & 75,498 \\
\hline \multirow{3}{*}{ RE } & $1(2,3)$ & 77,415 & 3,005 & 71,419 & 83,412 \\
\hline & $2(1)$ & 90,821 & 1,739 & 87,351 & 94,291 \\
\hline & $3(1)$ & 90,217 & 1,668 & 86,890 & 93,545 \\
\hline \multirow{3}{*}{ RS } & $1(2,3)$ & 69,112 & 3,481 & 62,165 & 76,059 \\
\hline & $2(1)$ & 86,957 & 2,271 & 82,425 & 91,488 \\
\hline & $3(1)$ & 82,065 & 2,700 & 76,677 & 87,454 \\
\hline \multirow{3}{*}{ SM } & $1(2,3)$ & 56,920 & 2,301 & 52,328 & 61,513 \\
\hline & $2(1)$ & 75,362 & 2,141 & 71,089 & 79,635 \\
\hline & $3(1)$ & 72,391 & 2,396 & 67,611 & 77,172 \\
\hline \multirow{3}{*}{ VT } & $1(2,3)$ & 52,355 & 2,316 & 47,734 & 56,976 \\
\hline & $2(1)$ & 71,558 & 2,259 & 67,050 & 76,066 \\
\hline & $3(1)$ & 69,112 & 2,421 & 64,282 & 73,942 \\
\hline
\end{tabular}


SA

\begin{tabular}{|l|l|l|l|l|}
\hline $1(2,3)$ & 48,188 & 1,953 & 44,291 & 52,086 \\
\hline $2(1)$ & 73,551 & 2,057 & 69,446 & 77,656 \\
\hline $3(1)$ & 76,812 & 3,067 & 70,691 & 82,933 \\
\hline
\end{tabular}

Tabla 2. Resultados de las tres evaluaciones para las distintas variables correspondientes a la Cuestionario SF-36. En la columna evaluación, distinto número indica diferencias significativas entre ambas, todas ellas menores de $p=0,050$. CSFIS: componente salud física, CSMEN: componente salud mental, CSTOT: componente salud total, FFIS: función física, LRF: rol físico, SG: salud general, LRE: rol emocional, LRS: rol social, SM: salud mental, V: vitalidad, SA: salud actual.

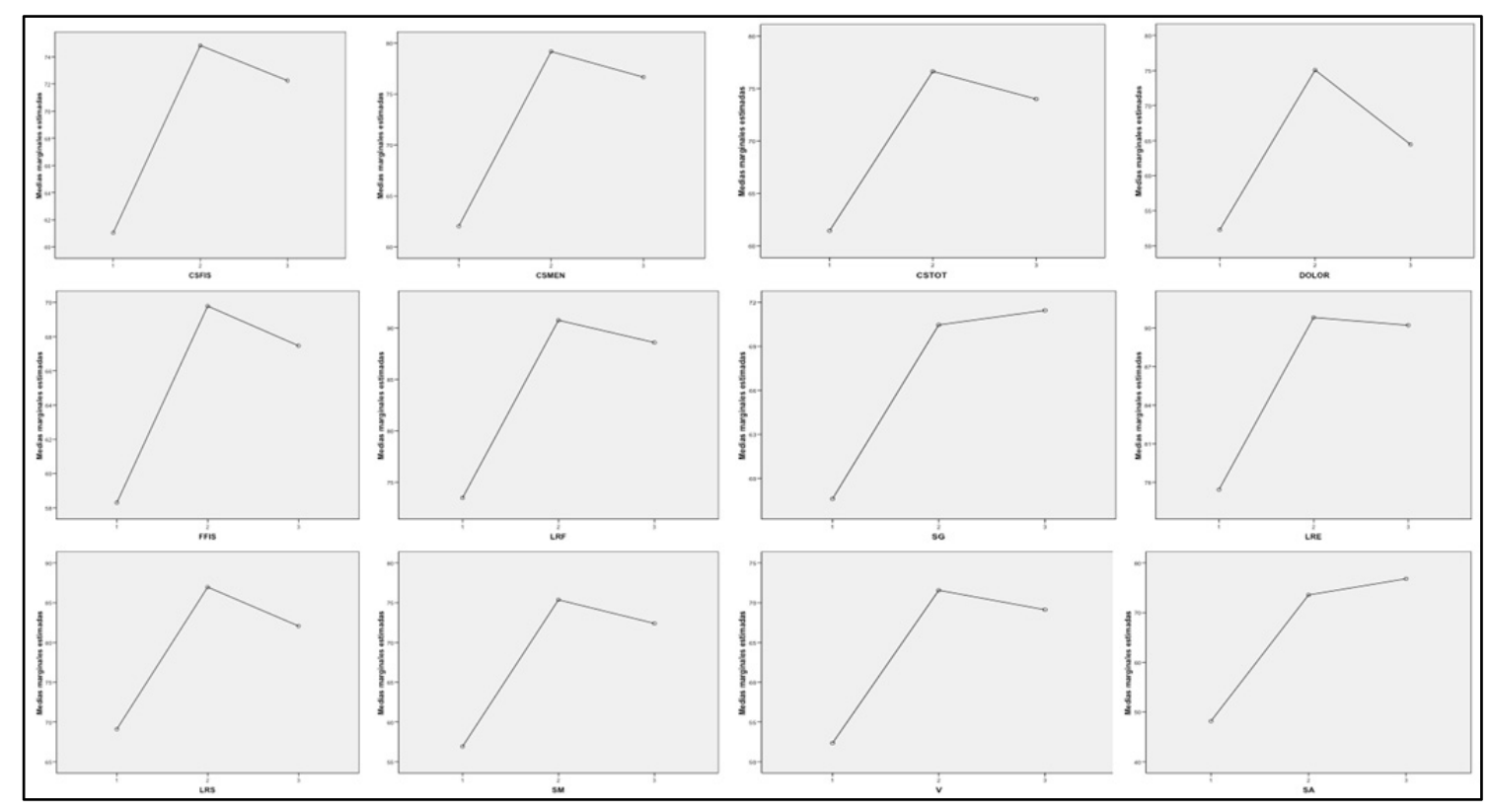

Figura 3. Representación gráfica de las tres tomas para las distintas variables correspondientes al Cuestionario de Salud SF-36v2. CSFIS: componente salud física, CSMEN: componente salud mental, CSTOT: componente salud total, FFIS: función física, LRF: rol físico, SG: salud general, LRE: rol emocional, LRS: rol social, SM: salud mental, V: vitalidad, SA: salud actual.

\section{DISCUSIÓN}

Son numerosos los trabajos centrados en programas de entrenamiento en adultos, proponiendo diferentes contenidos, pero poco se ha avanzado en el conocimiento del medio acuático sobre esta población. En esta misma línea, varios estudios han confirmado la eficacia que tiene la práctica de actividad física en el agua con respecto a la pérdida de peso, pérdida de grasa y ganancia de músculo, siendo esta última la más marcada con respecto a personas que practican actividad física en tierra $(15,16,17)$, lo que supone un hallazgo interesante que no debería ser pasado por alto.

La variable estado de ánimo de los participantes evidenció un aumento del vigor y una disminución de la fatiga, la hostilidad, la depresión y la tensión, coincidiendo la reducción de las dos últimas dimensiones con otros estudios . De ahí que la participación de los mayores en programas colectivos de actividad física sea de interés por su repercusión sobre su salud integral. 
Para las variables de percepción de salud encontramos que la actividad física en tierra mejora en todas sus variables $(19,20)$, al igual que refieren otros estudios en agua $(21,22)$. En nuestro estudio, los participantes mejoraron su percepción de salud al ser comparada con la del año anterior en las distintas evaluaciones. Para el resto de variables, CSM, CSF y CSTOT, se experimentó una mejora significativa durante las primeras 10 semanas y una tendencia a la baja durante las siguientes 10 semanas, sin llegar a los valores iniciales. Algo similar a lo que ocurre con las variables fisiológicas y donde podemos argumentar efectos similares como consecuencia de los procesos de adaptación en las primeras semanas.

\section{CONCLUSIONES}

Un programa de actividad física en el agua de 20 semanas de duración mejora la percepción del estado de ánimo de los participantes, produciéndose un aumento del vigor y una disminución de la hostilidad, la depresión, la tensión y la fatiga.

La actividad física realizada en grupo en el medio acuático tiene un efecto positivo sobre la percepción de la salud de los adultos mayores, tanto en el componente físico como en el mental.

\section{REFERENCIAS}

1. Lama, J. Biología del envejecimiento. In Geriatría. 2013. (p. 13). Medellín. Colombia: Corporación para Investigaciones Biológicas.

2. López, J. H. Fisiología del envejecimiento. In Geriatria. 2006. (pp. 17-22). Medellín, Colombia: Corporación para las investigaciones Biológicas.

3. Jiménez, M. Efectos que tiene un programa de actividad física dirigida sobre los niveles plamáticos de lípidos en mujeres de 60 (Tesis Doctoral). 2007. Universidad de Jaén, Jaén.

4. Saxon, S. V. Physical change \& aging: a guide for the helping professions (5th ed.). 2010. New York: Springer Pub. Co.

5. Millán, J. C. Gerontología: valoración e intervención. 2011. Madrid: M Panamericana.

6. Organización Mundial de la Salud [OMS]. Hombres, Envejecimiento y Salud: Conservar la salud a lo largo de la vida. 2001. OMS.

7. Organización Mundial de la Salud [OMS]. Actividad física. OMS. 2013. Retrieved October 31, 2013, from http://www.who.int/dietphysicalactivity/pa/es/

8. Organización Mundial de la Salud [OMS]. OMS | Enfermedades cardiovasculares. OMS. 2013. Retrieved November 25, 2013, from http://www.who.int/mediacentre/ factsheets/fs317/es/

9. Vilagut, G., Ferrer, M., Rajmil, L., Rebollo, P., Permanyer-Miralda, G., Quintana, J. M., Alonso, J. El Cuestionario de Salud SF-36 español: una década de experiencia y nuevos desarrollos. Gaceta Sanitaria 2005, 19(2), 135-150. 
10. Mate, F., \& Moya, A. Taller: programa de intervención en el medio acuático. In III Congreso Internacional de Actividad Físico Deportiva para Mayores. 2009. Málaga: CEDMA, Consejería de Turismo, Comercio y Deporte. Instituto Andaluz del deporte.

11. Soler, A. La psicomotricidad. Una propuesta eficaz para la dinamización corporal de las personas mayores. Lecciones de Gerontología. 2006. VI No. 58. (p. 20). Madrid: Portal Mayores.

12. Sanders, M. E. H20 solutions for active aging. Www. ideafit.com. 2010. 7(2).

13. American College of Sport Medicine. ACSM's Guidelines for Exercise Testing and Prescription 9th Ed. J Can Chiropr Assoc. 2014; 58(3): 328.

14. Andrade, E. L., Matsudo, S. M., Matsudo, V. R., \& Araújo, T. L. Body Mass Index and Neuromotor Performance in Active and Sedentary Elderly Women 880. Medicine \& Science in Sports \& Exercise. 1997. 29(5).

15. Gubani, G., Cândido, N., Petroski, L., \& da Silva, A. Efeitos da hidroginástica sobre indicadores antropométricos de mulheres enetre 60 e 80 añosde idade. Revista Brasileira de Cineantropometria \& Desempenho Humano. 2001. (1).

16. Nagle, E., Robertson, R., Jakicic, J., Otto, A., Ranalli, J., \& Chiapeta, L. Effects of aquatic exercise and walking in sedentary obese women undergoing a behavioral weight-loss intervention. Human Kinetics Journals. 2007. 1, 43-56.

17. Greene, N. P., Lambert, B. S., Greene, E. S., Carbuhn, A. F., Green, J. S., \& Crouse, S. F. Comparative efficacy of water and land treadmill training for overweight or obese adults. Medicine and Science in Sports and Exercise. 2009. 41(9), 1808-1815.

18. Katsura, Y., Yoshikawa, T., Ueda, S.-Y., Usui, T., Sotobayashi, D., Nakao, H., Fujimoto, S. Effects of aquatic exercise training using water-resistance equipment in elderly. European Journal of Applied Physiology. 2009. 108(5), 957-964.

19. Martín, M. Influencia de un programa de actividad física sobre aspectos físicos y psicológicos en personas de más de 55 años en la población del Algarve (Tesis Doctoral). Universitat de València, Servei de Publicacions. 2007. València.

20. Marques, J. M. Organização e gestão de um programa de exercício físico multicomponente : efeitos no custo com medicação, aptidão física, imunidade, perfil metabólico, estado de humor e qualidade de vida em idosos (Tesis Maestría). 2011. Universidad Coimbra.

21. Devereux, K., Robertson, D., \& Briffa, N. Effects of a water-based program on women 65 years and over: A randomised controlled trial, 2005. 51, 102-108.

22. Freitas, M. Qualidade de vida associada à saúde com efeitos psicológicos da atividade física em idosas praticantes de hidroginástica. Efdeportes.com, 2011.152.

Total referencias bibliográficas: 22

Referencias citadas correspondientes a RICCAFD: 0 